\title{
Photodynamic Therapy in Unresectable Cholangiocarcinoma: Not for the Uncommitted
}

\author{
Jayant P. Talreja ${ }^{1}$, Marisa DeGaetani ${ }^{2}$, Kristi Ellen ${ }^{1}$, Timothy Schmitt ${ }^{1}$, Monica Gaidhane ${ }^{2}$ and \\ Michel Kahaleh ${ }^{2}$
}

1'Department of Gastroenterology and Hepatology, University of Virginia School of Medicine, Charlottesville, VA, ${ }^{2}$ Division of Gastroenterology and Hepatology, Department of Medicine, Weill Cornell Medical College, New York, NY, USA

Background/Aims: Photodynamic therapy (PDT) in unresectable cholangiocarcinoma has been associated with improved survival. We report a single tertiary care center experience over the past 6 years.

Methods: Fifty-five patients with unresectable cholangiocarcinoma received PDT between 2004 and 2010. Plastic stents were placed after PDT to prevent cholangitis.

Results: Twenty-seven patients (49\%) showed Bismuth type IV, 22 (41\%) showed Bismuth type III, and six (10\%) showed Bismuth type I and II. Twenty patients (37\%) received chemotherapy and radiation therapy, five (9\%) received chemotherapy only; and one (2\%) received radiation therapy only. Mean number of PDT sessions was $1.9 \pm 1.5$ sessions (range, 1 to 9). Mean survival duration was $293 \pm 266$ days (median, 190; range, 25 to 1,332). PDT related complications included three (5\%) facial burn, three (5\%) photosensitivity, and two (3\%) rash. Kaplan-Meier analysis comparing the survival means of patients who received PDT and chemotherapy/radiation therapy (median survival 257 days; 95\% confidence interval [CI], 166 to 528) versus who received PDT only (median survival 183 days; $95 \%$ CI, 129 to 224 ) showed no significant difference (log-rank $p=0.20$ ).

Conclusions: PDT has a measurable impact on survival in unresectable cholangiocarcinoma but requires aggressive stenting posttherapy.

Key Words: Photodynamic therapy; Cholangiocarcinoma; Single operator choledochoscopy

\section{INTRODUCTION}

Cholangiocarcinoma, a malignancy that arises from the epithelial cells of the biliary tree, causes significant morbidity and mortality, with an average 5 -year survival rate of $5 \%$ to $10 \%{ }^{1}$ Cure is achieved only through surgical resection; yet, greater than $80 \%$ of patients present at an advanced and unresectable stage, ${ }^{2}$ resulting in a 3- to 6-month median survival from the time of diagnosis. ${ }^{3}$ Various palliative treatment strategies have been offered, including chemotherapy, radiation therapy and biliary drainage (endoscopic or percutaneous), but none have

Received: October 17, 2012 Revised: January 10, 2013

Accepted: February 28, 2013

Correspondence: Michel Kahaleh

Division of Gastroenterology and Hepatology, Department of Medicine, Weill Cornell Medical College, 4th Floor, 1305 York Ave, New York, NY 10021, USA Tel: +1-646-962-4000, Fax: +1-646-962-0110, E-mail: mkahaleh@gmail.com

(c) This is an Open Access article distributed under the terms of the Creative Commons Attribution Non-Commercial License (http://creativecommons.org/ licenses/by-nc/3.0) which permits unrestricted non-commercial use, distribution, and reproduction in any medium, provided the original work is properly cited. shown a definitive survival benefit. ${ }^{2,3}$ Photodynamic therapy (PDT) is a promising new treatment modality for nonresectable cholangiocarcinoma. Its benefits include symptomatic improvement and prolonged survival with relatively few complications. $^{2}$

With PDT, a photosensitizing agent is preferentially retained by the malignant tissue. Following laser activation at a distinct wavelength, toxic oxygen radicals induce apoptosis of malignant cells and cause tumor necrosis. ${ }^{2-4}$ PDT has been reported to have a minimal side effect profile, with the most frequently encountered adverse effect being phototoxicity to the skin. $^{2}$

In the last few years we have seen the rise of cholangioscopyguided PDT, which allows better identification of tumor margins, permits targeted laser illumination, and aids in evaluating the response to therapy. ${ }^{5,6}$ We now report our experience with PDT, in the treatment of cholangiocarcinoma over the past 6 years. 


\section{MATERIALS AND METHODS}

\section{Patients}

Fifty-five patients (31 males, 24 females) with unresectable cholangiocarcinoma received treatment with PDT between 2004 and 2010. Patient characteristics are summarized in Table 1 . The median age was 69 years (range, 30 to 87 ). Twentyseven patients (49\%) showed Bismuth type IV, 22 (41\%) showed Bismuth type III, and six (10\%) showed Bismuth type I and II. The median median model for end-stage liver disease score was 15 (range, 7 to 25 ). Twenty patients (37\%) received both chemotherapy and radiation therapy. Five patients (9\%) received chemotherapy only and one patient (2\%) received radiation therapy only.

Statistical analysis was conducted using SAS 9.2 (SAS Institute Inc., Cary, NC, USA). Kaplan-Meier analysis was performed comparing the group of patients who received PDT and chemotherapy/radiation therapy versus those who received PDT only.

\section{Procedure}

Photofrin (porfirmer sodium) $2 \mathrm{mg} / \mathrm{kg}$ body weight was administered intravenously 48 hours prior to irradiation. PDT was delivered through a $3.0-\mathrm{m}$ length fiber with a 2.5 -cm-long cylindrical diffuser at its distal end (Pioneer Optics, Windsor Locks, CT, USA) (Fig. 1). Single operator cholangioscopy (SOC) was utilized for fiber placement in 18 patients (Figs. 2, 3). Photoactivation was performed at $630 \mathrm{~nm}$ with a light dose of

Table 1. Patient Characteristics

\begin{tabular}{lc}
\multicolumn{1}{c}{ Characteristic } & Value \\
\hline No. of patients & 55 \\
Gender, male/female & $31 / 24$ \\
Median age, yr & $69(30-87)$ \\
Bismuth classification & \\
Bismuth IV & $27(49)$ \\
Bismuth III & $22(41)$ \\
Bismuth I and II & $6(10)$ \\
MELD score & $15(7-25)$ \\
Treatment group 1, subjects & \\
PDT+chemotherapy+radiation therapy & $26(26 / 55)$ \\
Chemotherapy+radiation therapy & $20(37)$ \\
Chemotherapy only & $5(9)$ \\
Radiation therapy only & $1(2)$ \\
Treatment group 2, subjects & \\
PDT therapy only & $29(29 / 55)$ \\
\hline
\end{tabular}

Values are presented as median (range) or number (\%).

MELD, median model for end-stage liver disease; PDT, photodynamic therapy.
$180 \mathrm{~J} / \mathrm{cm}^{2}$, fluence of $0.250 \mathrm{~W} / \mathrm{cm}^{2}$, and irradiation time of 750 seconds.

The single operator cholangioscope was inserted into the bile duct over the wire. Direct visualisation of the cancerous stricture was conducted macroscopically which permitted exchange of the wire for the diffuser, which was centered within the stricture and allowed target administration of light.

Placement of plastic stents was performed systematically after the photodynamic treatment to prevent cholangitis. All patients received perioperative antibiotic prophylaxis.

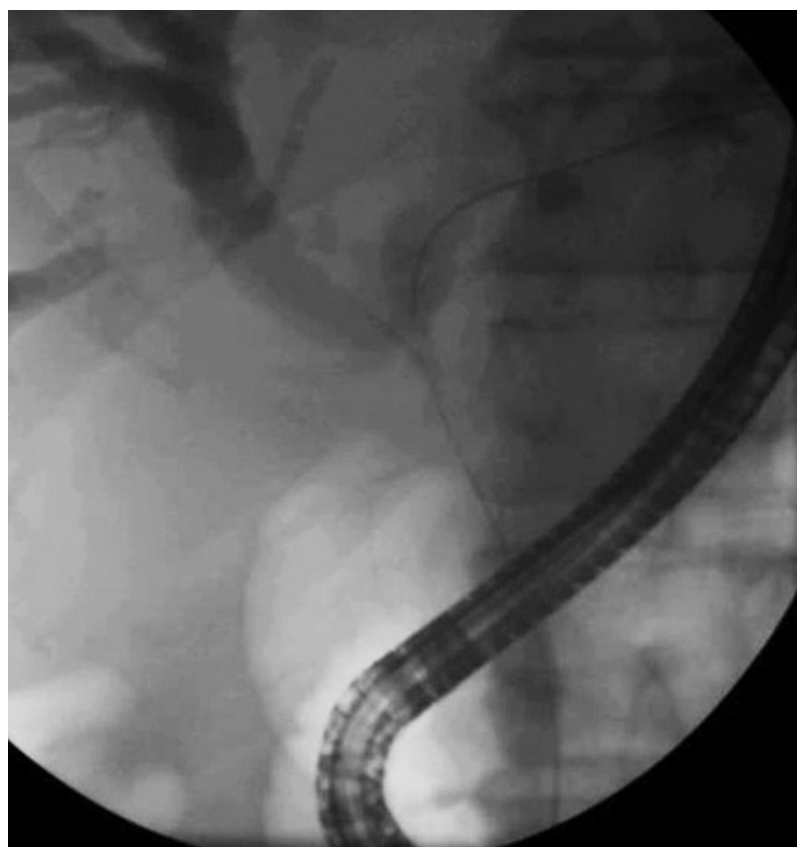

Fig. 1. Fluoroscopy demonstrating malignant stricture involving the confluence.

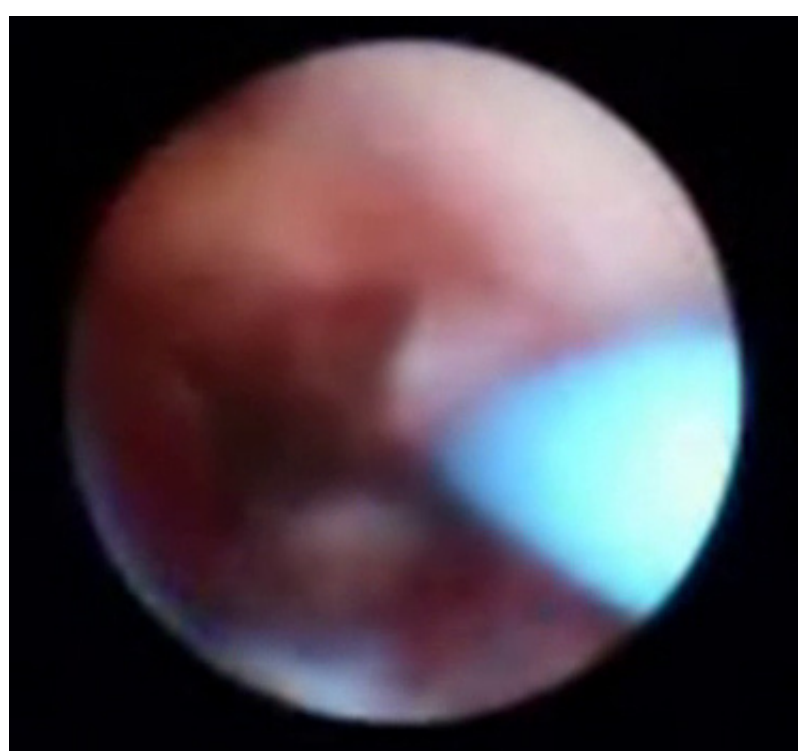

Fig. 2. Single operator choledochoscope placed below the stricture to be treated. 

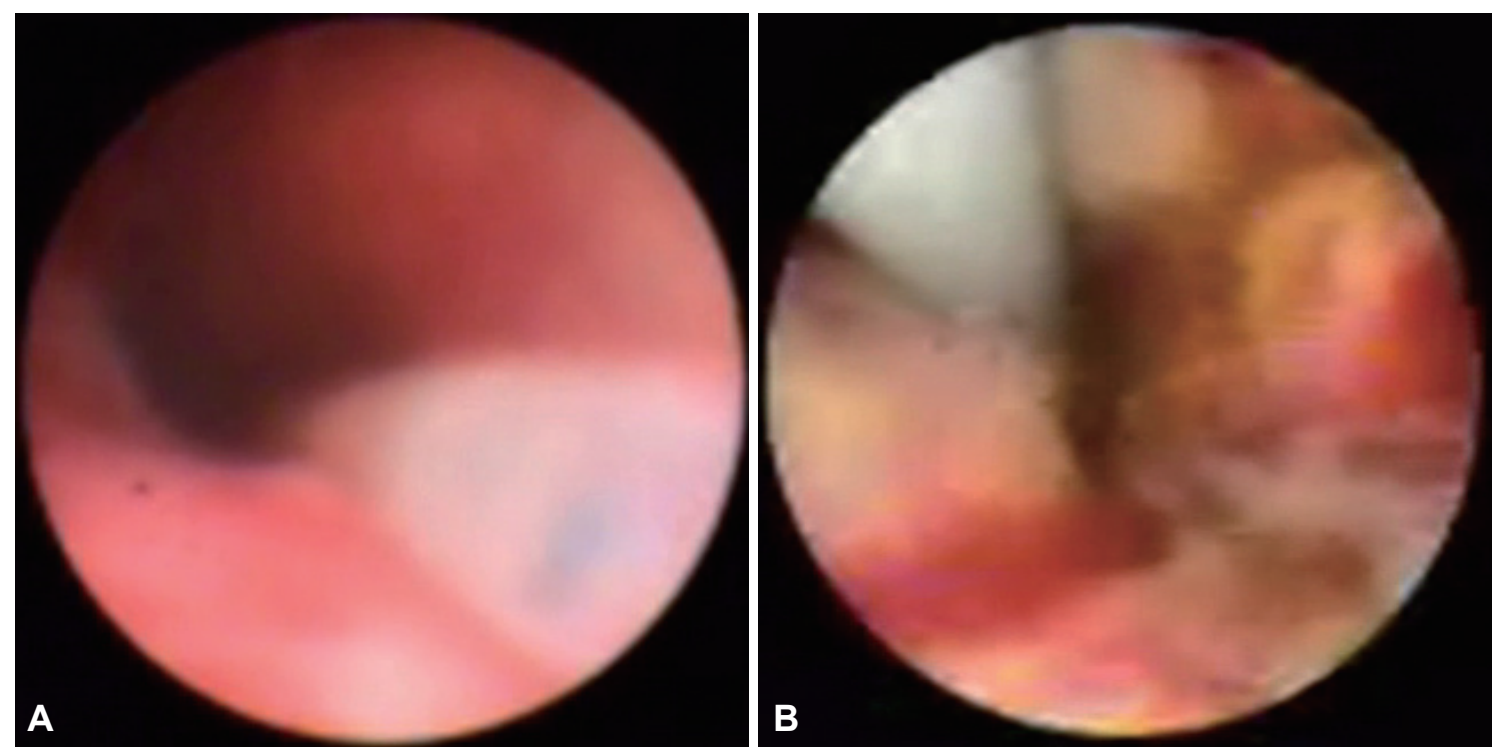

Fig. 3. (A) Choledochoscopy after centering the fiber diffuser at the level of the stricture. (B) Choledochoscopy after 3 months postphotodynamic therapy.

\section{RESULTS}

Fifty-five patients (31 males, 24 females) with unresectable cholangiocarcinoma received treatment with PDT between 2004 and 2010. Twenty-five patients received chemotherapy with various combinations of gemcitabine and capecetabine; and one received radiation therapy only along with PDT. Twenty-nine patients received PDT only.

The median age was 69 years (range, 30 to 87). Results are summarized in Table 2 . The median interval between presentation and first PDT session was 63 days and there was a median follow-up of 133 days (range, 11 to 1,190). The mean number of PDT sessions was $1.9 \pm 1.5$ sessions (median, 1 ; range, 1 to 9). There was a mean survival of $293 \pm 266$ days (median, 190 ; range, 25 to 1,332). Median survival of patients who received PDT and chemotherapy/radiation therapy was 257 days ( $95 \%$ confidence interval [CI], 166 to 528) while medial survival duration of the PDT only group was 183 days (95\% CI, 129 to 224).

During follow-up, 49/55 patients died from progression or complications of their underlying disease. There were no procedure related deaths.

Twenty-eight (51\%) developed interval cholangitis requiring stent revision. All of these patients showed Bismuth type III and IV lesions. Other non-PDT related complications included: six (11\%) hepatic abscess, one (2\%) gallbladder perforation, two (3\%) peritonitis, related to systemic infection, two (3\%) pancreatitis, two (3\%) hemobilia, and three (5\%) cholecystitis. Eight patients experienced PDT related adverse events, including the following: three (5\%) facial burn, three (5\%) photosensitivity, and two (3\%) rash.

Kaplan-Meier analysis (Fig. 4) was performed comparing
Table 2. Complications

\begin{tabular}{lc}
\hline & No. (\%) \\
\hline Non-PDT related complications & \\
Cholangitis & $28(51)$ \\
Hepatic abscess & $6(11)$ \\
Gallbladder perforation & $1(2)$ \\
Peritonitis & $2(3)$ \\
Pancreatitis & $2(3)$ \\
Hemobilia & $2(3)$ \\
Cholecystitis & $3(5)$ \\
PDT related complications & \\
Facial burn & $3(5)$ \\
Photosensitivity & $3(5)$ \\
Rash & $2(3)$ \\
\hline
\end{tabular}

PDT, photodynamic therapy.

the group of patients who received PDT and chemotherapy/ radiation therapy versus those who received PDT only.

After adjusting for age, sex, and Bismuth classification, there was no significant difference in the survival means of both groups $(\log$-rank $p=0.20)$.

\section{DISCUSSION}

Palliative therapy is the mainstay of treatment in patients who present with unresectable cholangiocarcinoma. Biliary decompression, which is usually accomplished via endoscopic stent placement, can result in symptom improvement and prevent complications such as cholangitis and sepsis; however, stent placement has not been shown to have any survival benefit $^{2}$ and is limited by recurrent stent occlusion. Additionally, 

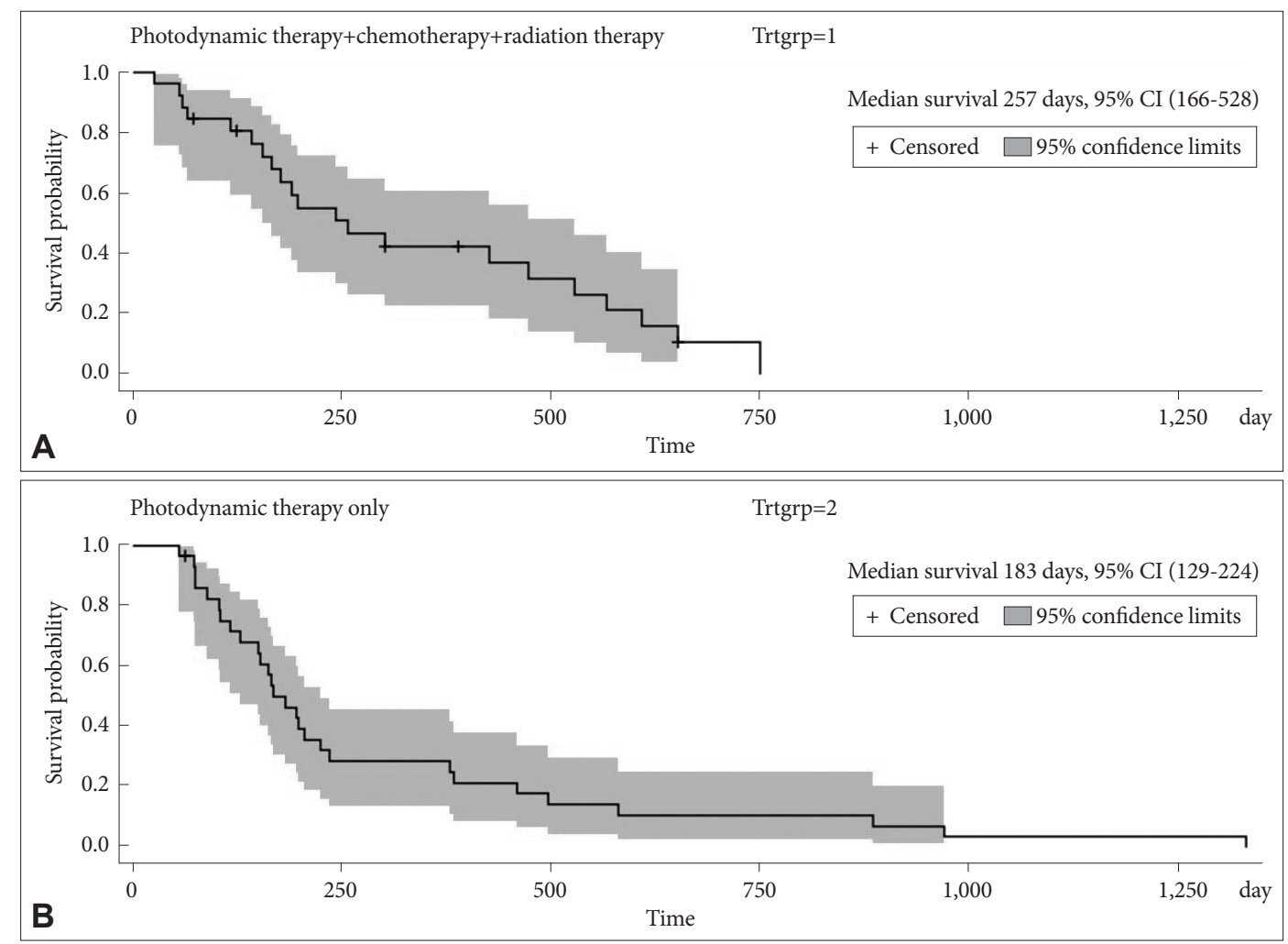

Fig. 4. Kaplan-Meier analysis. (A) Photodynamic therapy+chemotherapy+radiation therapy. (B) Photodynamic therapy only. Cl, confidence interval.

successful drainage through stent placement proves to be more difficult in proximal tumors, ${ }^{7}$ necessitating the search for a better treatment modality.

Numerous studies, including two randomized clinical trials, have found that PDT is superior to biliary stenting alone in the treatment of unresectable cholangiocarcinoma, improving both survival as well as quality of life. ${ }^{2,3,8-13}$ Additionally, it was recently shown that survival in patients who underwent PDT was similar to those in whom curative surgery was attempted but who had positive resection margins, despite the fact that the PDT group had more advanced clinical T stages. ${ }^{14}$

Utilization of cholangioscopy with PDT allows direct visualization of the biliary tree, leading to improvements in diagnosis and treatment. It also has been shown to influence decisions regarding patient management. ${ }^{5,6}$ Our group compared PDT versus SOC directed PDT and found the therapies to be comparable without an increase in adverse complications, but with decreased radiation exposure in the SOC-directed group. ${ }^{15}$ Another recent study demonstrated that direct POCguided PDT using an ultraslim upper endoscope is both feasible and safe in select patients with inoperable extrahepatic cholangiocarcinoma. ${ }^{16}$

Our center's cumulative experience with PDT for the treatment of unresectable cholangiocarcinoma, which is one of the largest studies to date, again demonstrated its survival bene- fit, with a mean survival of $293 \pm 266$ days (median, 190; range, 25 to 1,332). No difference in survival times was noted between those treated with PDT alone and those with PDT and chemo or radiation therapy. However, despite its benefit, PDT was also associated with a substantial incidence of cholangitis requiring stent revision, occurring in just over $50 \%$ of our patients. A review of 20 studies of PDT in cholangiocarcinoma found the overall incidence of cholangitis to be $27.5 \%,{ }^{2}$ with the largest clinical trial showing an incidence of $56 \% .{ }^{17}$ Another recent study showed a 57\% incidence of cholangitis with PDT. ${ }^{12}$ Although cholangitis can be a complication of stenting itself, seen in $20 \%$ to $40 \%$ of cases using plastic stents and $4.9 \%$ to $6 \%$ of cases using bare metal stents, ${ }^{18}$ the high rate of cholangitis post-PDT is likely the result of necrosis associated with this therapy. Given the frequency with which this complication occurs, aggressive stenting posttherapy is required. This is a commitment that both physician and patient must be aware of before commencing therapy.

In conclusion, use of PDT for the treatment of unresectable cholangiocarcinoma is associated with increased survival compared to stenting alone. However, PDT therapy is associated with high rates of cholangitis and stent revision, indicating the need for aggressive stenting posttherapy. A prospective multicenter randomized controlled trial is needed to confirm the benefit of PDT with stenting versus stenting alone as 
well as the benefit of PDT plus chemotherapy and radiation therapy compared with PDT alone.

\section{Conflicts of Interest}

The authors have no financial conflicts of interest.

\section{Acknowledgments}

Data presented in this manuscript has been collected from an Institutional Review Board (IRB) Approved database protocol (IRB \#11325 ERCP in Pancreatico-biliary Disease; Original Approval on 2004 May 24).

\section{REFERENCES}

1. Anderson CD, Pinson CW, Berlin J, Chari RS. Diagnosis and treatment of cholangiocarcinoma. Oncologist 2004;9:43-57.

2. Gao F, Bai Y, Ma SR, Liu F, Li ZS. Systematic review: photodynamic therapy for unresectable cholangiocarcinoma. J Hepatobiliary Pancreat Sci 2010;17:125-131.

3. Wiedmann M, Berr F, Schiefke I, et al. Photodynamic therapy in patients with non-resectable hilar cholangiocarcinoma: 5-year follow-up of a prospective phase II study. Gastrointest Endosc 2004;60:68-75.

4. Zoepf T. Photodynamic therapy of cholangiocarcinoma. HPB (Oxford) 2008;10:161-163

5. Judah JR, Draganov PV. Intraductal biliary and pancreatic endoscopy: an expanding scope of possibility. World J Gastroenterol 2008;14:31293136.

6. Fishman DS, Tarnasky PR, Patel SN, Raijman I. Management of pancreaticobiliary disease using a new intra-ductal endoscope: the Texas experience. World J Gastroenterol 2009;15:1353-1358.

7. Cheon YK. The role of photodynamic therapy for hilar cholangiocarcinoma. Korean J Intern Med 2010;25:345-352.

8. Zoepf T, Jakobs R, Arnold JC, Apel D, Riemann JF. Palliation of nonresectable bile duct cancer: improved survival after photodynamic therapy. Am J Gastroenterol 2005;100:2426-2430.
9. Ortner ME, Caca K, Berr F, et al. Successful photodynamic therapy for nonresectable cholangiocarcinoma: a randomized prospective study. Gastroenterology 2003;125:1355-1363.

10. Kahaleh M, Mishra R, Shami VM, et al. Unresectable cholangiocarcinoma: comparison of survival in biliary stenting alone versus stenting with photodynamic therapy. Clin Gastroenterol Hepatol 2008;6:290-297.

11. Harewood GC, Baron TH, Rumalla A, et al. Pilot study to assess patient outcomes following endoscopic application of photodynamic therapy for advanced cholangiocarcinoma. J Gastroenterol Hepatol 2005;20: 415-420.

12. Fuks D, Bartoli E, Delcenserie R, et al. Biliary drainage, photodynamic therapy and chemotherapy for unresectable cholangiocarcinoma with jaundice. J Gastroenterol Hepatol 2009;24:1745-1752.

13. Quyn AJ, Ziyaie D, Polignano FM, Tait IS. Photodynamic therapy is associated with an improvement in survival in patients with irresectable hilar cholangiocarcinoma. HPB (Oxford) 2009;11:570-577.

14. Matull WR, Dhar DK, Ayaru L, et al. R0 but not R1/R2 resection is associated with better survival than palliative photodynamic therapy in biliary tract cancer. Liver Int 2011;31:99-107.

15. Talreja JP, Degaetani M, Sauer BG, Kahaleh M. Photodynamic therapy for unresectable cholangiocarcinoma: contribution of single operator cholangioscopy for targeted treatment. Photochem Photobiol Sci 2011; 10:1233-1238.

16. Choi HJ, Moon JH, Ko BM, et al. Clinical feasibility of direct peroral cholangioscopy-guided photodynamic therapy for inoperable cholangiocarcinoma performed by using an ultra-slim upper endoscope (with videos). Gastrointest Endosc 2011;73:808-813.

17. Witzigmann H, Berr F, Ringel U, et al. Surgical and palliative management and outcome in 184 patients with hilar cholangiocarcinoma: palliative photodynamic therapy plus stenting is comparable to $\mathrm{r} 1 / \mathrm{r} 2$ resection. Ann Surg 2006;244:230-239.

18. Guglielmi A, Ruzzenente A, Iacono C. Surgical Treatment of Hilar and Intrahepatic Cholangiocarcinoma. Verona (IT): Springer; 2008. 